\title{
DESCRIPCIÓN ANATÓMICA DE LOS MÚSCULOS DEL MIEMBRO POSTERIOR Y COLA DEL MONO MACHIN BLANCO (Cebus albifrons)
}

\author{
Anatomical Description of Muscles in the Hind Limb and Tail of Cebus \\ ALBIFRONS
}

\author{
Miriam Quevedo U. ${ }^{1}$, Jannet Cisneros S. ${ }^{1}$, Miluska Navarette Z. ${ }^{1}$, Juan Pablo Torres \\ G. ${ }^{1}$ y Alberto Sato $S^{1,2}$
}

\section{Resumen}

El presente estudio tuvo como objetivo realizar la descripción anatómica de los músculos del miembro posterior y cola del mono Machín Blanco (Cebus albifrons). Se utilizaron 4 monos castrados, adultos, con más de 3 años. Los monos fueron eutanasiados y embalsamados, y luego de 6 días se procedió a la divulsión de los miembros posteriores y la cola, describiéndose todos los músculos cutáneos superficiales y profundos, tomando como referencia estudios previos en el mono Rhesus (Maccaca mullata). Las regiones de la pelvis y muslo, pierna y pie presentaron 18, 12 y 11 músculos, respectivamente. No se encontró el músculo piriforme, peroneotibial, peróneo digital del IV dedo y el oponente del primer dedo. En la cola se hallaron 10 músculos, número mayor a lo encontrado en el mono Rhesus.

Palabras clave: Cebus albifrons, músculo, miembro posterior, cola

\section{AbSTRaCt}

The objective of this study was to perform the anatomic description of the muscles located in the hind limb and tail of Cebus albifrons. Four adult monkeys older than 3 years of age and castrated were used. The animals were euthanized and embalmed; then, after six days, the dissection of the hind limb and tail was carried out. Muscles were described in comparison with the Rhesus monkey (Maccaca mullata). The pelvis and thigh, leg and foot region presented 18,12 and 11 muscles respectively. Piriformis, peroneotibialis, peroneus digiti quarti and the opponens digiti hallucis muscles were not present. The tail region presented 10 muscles, a higher number as compared to the Rhesus.

Key words: Cebus albifrons, muscle, hind limb, tail

\footnotetext{
${ }^{1}$ Laboratorio de Anatomía y Fauna Silvestre, Facultad de Medicina Veterinaria, Universidad Nacional Mayor de San Marcos, Lima

2 E-mail: albertosato2000@yahoo.com
} 
Los primates actualmente se clasifican en "Monos del Viejo Mundo" y "Monos del Nuevo Mundo" distribuyéndose los primeros en África y Asia, principalmente; mientras que los últimos tienen en Centro y Sudamérica sus principales áreas de distribución (Arsuaga y Martínez ,1998). Dentro de los "Monos del Nuevo Mundo" se encuentran múltiples especies, las cuales poseen principalmente características arbóreas, a diferencia de los "Monos del Viejo Mundo", cuyo desplazamiento se desarrolla mayormente en el suelo.

Dentro de las especies que viven en Sudamérica, se encuentra el Cebus albifrons, llamado comúnmente Machín Blanco (Fortman et al., 2002), especie que no está en situación de amenaza, pero su población podría disminuir si no se controla su comercio ilegal, así como la fuerte presión de caza como ocurre en el Perú (Hershkovitz, 1977).

Existen pocos estudios realizados en relación a la musculatura que controla sus movimientos, habiéndose, en cambio, desarrollado investigaciones más detalladas sobre la anatomía de los "Monos del Viejo Mundo", como es el caso del mono Rhesus (Maccaca mulatta) (Aversi, 2004). El miembro posterior y cola son la base de la locomoción de primates de Sudamérica, ya que son principalmente arbóreos y requieren mantener un excelente equilibrio en los árboles. Por ello, la investigación en el Cebus albifrons se hace necesaria en el Perú, a fin de obtener información que contribuya al manejo eficiente de los primates no humanos.

En el presente trabajo se realizó una descripción detallada de la musculatura de los miembros posteriores y cola en especímenes de la especie Cebus albifrons, determinando sus relaciones respecto a la anatomía de "Monos del Viejo Mundo", principalmente del mono Rhesus.
El presente estudio se realizó en el Laboratorio de Anatomía Animal y Fauna Silvestre de la Facultad de Medicina Veterinaria de la Universidad Nacional Mayor de San Marcos (FMV-UNMSM), Lima. Se emplearon cuatro ejemplares de monos machines blancos (C. albifrons), machos adultos (3 años) castrados, donados por el Patronato Parque de las Leyendas.

Los animales fueron anestesiados (10$15 \mathrm{mg} / \mathrm{kg}$ ketamina y $3 \mathrm{mg} / \mathrm{kg}$ xilacina) y sacrificados con solución de sulfato de sodio concentrado. Los cadáveres fueron trasladados a la FMV-UNMSM, donde se realizó el proceso de embalsamamiento. Luego de 6 días de formalizados, se procedió a la divulsión de los miembros posteriores y de la cola para realizar la identificación y descripción de los músculos cutáneos, superficiales y profundos de acuerdo a la Nómina Anatómica Veterinaria (ICVGAN, 2005) y a la terminología empleada en anatomía humana.

Al final, se realizó el registro fotográfico de los músculos, como ayuda visual para determinar las diferencias y semejanzas con los músculos descritos en el mono Rhesus (M. mulatta) por Bast et al. (1961).

\section{Resultados y Discusión}

Se identificaron 51 músculos en el miembro posterior y la cola, cifra ligeramente mayor en relación a los músculos del mono Rhesus, en cual se han descrito 50 músculos en estas dos regiones (Cuadro 1). La mayor diferencia en el número de músculos se observó a nivel de la cola, donde el Machín Blanco presentó 10 músculos en tanto que el macaco presenta 6 músculos (Bast et al., 1961).

La descripción anatómica de los músculos de la cadera y muslo de los especímenes divulsionados concordó en su mayoría con lo 
Cuadro 1. Número de músculos del miembro posterior y cola del Cebus albifrons vs. Maccaca mullata

\begin{tabular}{lcc}
\hline Área corporal & Cebus albifrons & ${\text { Maccaca } \text { mullata }^{1}}$ \\
\hline Muslo y pelvis & 18 & 19 \\
Pierna & 12 & 14 \\
Pie & 11 & 11 \\
Cola & 10 & 6 \\
\hline Total & 51 & 50 \\
\hline
\end{tabular}

${ }^{1}$ Fuente: Bast et al. (1961)

descrito en el mono Rhesus; sin embargo, se observaron algunas diferencias.

\section{Pelvis y muslo}

En esta región se encontró 18 músculos situados en dos planos: a) superficial craneal y medial, y b) profundo lateral y medial.

- $\quad$ No se encontró el músculo piriforme en el mono C. albifrons, que en compensación posee un grupo de músculos capaces de mover y agitar la cola hacia lateral y dorsoventral, así como deprimirla y enrollarla. En el muslo del mono Rhesus, este músculo está mezclado con las fibras del músculo glúteo medio, el cual es considerado como glúteo accesorio en otras especie de primates, debido a la falta de diferenciación del origen (Frey, 1993). Este músculo cumple la función de extender la articulación femoro-tibiorotuliana y agitar la cola (Bast et al., 1961).

- $\quad$ El músculo bíceps femoral, identificado en el C. albifrons, presentó una sola porción originado de la tuberosidad isquiática, y una amplia hoja conectada con fibras de la fascia lata, similar a lo descrito en el Machaca mulatta. En algunos mamíferos, el bíceps femoral presenta dos partes con separación completa o parcial (Bast et al., 1961).
- En el C. albifrons, el músculo semitendinoso presentó dos porciones: a) propia y b) accesorio, similar a lo descrito en el mono Rhesus. En el hombre, el músculo semitendinoso se encuentra unido al aductor mayor (adductor magnus ) formando un músculo compuesto (Frey,1993).

- En el C. albifrons, el músculo sartorio se ubicó en la cara medial del muslo y se originó a partir del borde acetabular del íleon. Estas características son similares a las del mono Rhesus (Bast et al., 1961).

\section{Pierna}

Se encontró 12 músculos localizados en los planos postero-medial y postero-lateral de la pierna, y en un esquema similar a lo descrito para el Maccaca mulatta. Esto incluye al músculo tibial, músculo flexor fibular, una hoja común flexora que se extiende hasta los dedos y una hoja profunda que pasa por el peroné y la tibia (Frey, 1993). Las principales observaciones fueron:

- El músculo tibial, representado por el gastrocnemio medial, y el músculo fibular, representado por el gastrocnemio lateral que dio origen a dos músculos adicionales: el músculo soleo y el músculo plantar, concuerda a lo descrito en 
el Maccaca mulatta (Frey 1993). En ciertos primates, el músculo soleo presenta una segunda cabeza tibial, como lo reportado en algunos monos antropoides y el hombre (Bast et al., 1961)

- El músculo plantar se encuentra en la mayor parte de mamíferos, así como en prosimios, monos (como el M. mullata) y el hombre, estando ausente en gibones, gorilas, orangutanes y chimpancés (Bast et al., 1961). En el hombre, su tamaño y extensión es variable, incluso, a veces, puede estar ausente. Su función, en los monos, es tensar la aponeurosis plantar y asistir en la flexión del pie, lo que ayuda a su desplazamiento (Bast et al., 1961). En el caso de los humanos su acción carece de importancia ( $\mathrm{O}^{\prime}$ Rahilly, 1989).

- El músculo peroneotibial (llamado también pronador tibial), no se encontró en el $C$. albifrons, debido a que su función es llevada a cabo por completo por el músculo poplíteo. El músculo peroneotibial, que se encuentra presente en muchos mamíferos como en el $M$. mullata, prosimios, gibones, en algunos casos grandes monos y el hombre, cumple la función de mantener la rodilla estable en la rotación de la tibia (Bast et al., 1961).

- En el grupo de los músculos flexores digitales, el flexor digital fibular del $C$. albifrons dio origen a tendones largos para el III y IV dedos que perforaron a los tendones del flexor digital corto, mientras que el flexor digital tibial dio origen a tendones largos para el II y V dedos y tendones cortos que se unieron a los tendones del flexor digital fibular del III y IV dedo del pie. En el mono M. mullata se localizan los músculos digitales fibulares y tibiales, que tienen un mismo origen. De cada uno se origina un tendón para cada dedo (Shuck, 1963). Dentro de las familias de monos, la distribución exacta de los tendones está sujeta a mucha variación (Frey, 1993).

- $\quad$ El músculo tibial craneal se encontró en el C. albifrons dividido en dos porciones longitudinales, siendo la porción medial más desarrollada que la lateral. Esta división ocurre en la mayoría de monos, incluyendo el M. mullata, pero la variabilidad está en el grado en la que el músculo se divide. Es decir, que puede dar origen a solo un tendón terminal o puede ser tan grande para separar el músculo entero en mitades longitudinales (Bast et al., 1961). En el hombre no se divide y tiene, por lo tanto, un solo tendón de inserción (O'Rahilly, 1989).

- Los músculos extensores peróneos digitales del M. mullata son dos, uno para el IV dedo y otro para el V dedo, cuya función es extender los dedos del pie. En los monos antropoideos y el hombre, el peróneo digital del IV dedo ha desaparecido, pero en primates inferiores se conserva la función (Frey, 1993). En el C. albifrons solo se identificó el peróneo digital del V dedo, el cual asume la función del peróneo digital del IV dedo.

Pie

En el pie se identificaron 11 músculos, los cuales fueron divididos en grupo dorsal y plantar:

- En el lado plantar, el músculo flexor digital corto presenta una cabeza superficial y una profunda. El número y distribución de los tendones de cada cabeza está sujeto a variación, como en el caso de muchos primates e incluso en el hombre. En el M. mullata y en primates primitivos (lemur, trásero y aye aye), la cabeza superficial proporciona un solo tendón para el II dedo y la cabeza profunda al III, IV y V dedo (Bast et al., 1961). En los "Monos del Viejo Mundo" como los gibones, orangutanes, gorilas y chimpancés, el tendón que se dirige al $\mathrm{V}$ 
dedo del pie está ausente por modificaciones de adaptación (Bast et al., 1961). En el caso del hombre, los cuatro tendones se originan de la cabeza superficial, mientras que la cabeza profunda, al estar presente, proporciona tendones laterales al V dedo del pie (O`Rahilly, 1989).

- El músculo oponente del I dedo (oppenens hallucis) no se encontró en el $C$. albifrons pero está descrito en el M. mullata. Este músculo permite oponer el I dedo del pie a los otros dedos (Bast et al., 1961).

- Los músculos interóseos, lumbricales y contrayentes que flexionan y extienden los dedos del pie se identificaron en los especímenes divulsionados. La orientación de los interóseos dorsales es similar a la distribución de los músculos en la mano, la cual toma como eje el tercer dedo (Bast et al., 1961). En el caso del pie del hombre, el segundo dedo es tomado como eje (Bast et al., 1961).

\section{Cola}

- La cola del Cebus albifrons tiene una longitud de 40.1 a $47.5 \mathrm{~cm}$, siendo de características prensiles (Hill, 1960) y considerada una extremidad más, a diferencia del M. mullata que mide de 19 a $32 \mathrm{~cm}$ y no es prensil (Jones et al., 2004). En los monos con cola prensil, las vértebras son más pequeñas dorsoventral y craneocaudalmente que en animales con cola no prensil; asimismo, existen más articulaciones y las vértebras están más juntas lo que le proporciona mayor flexibilidad y movimiento. Cuando los monos se cuelgan, el peso del cuerpo recae sobre el esqueleto axial, por lo que es fuerte y robusto. En el caso del M. mullata, ocurre lo contrario pues el tamaño y la separación de las vértebras es más grande y el peso del cuerpo recae sobre la pelvis cuando se cuelgan (Ankel-Simons, 2000).
- Se identificó 10 músculos en la cola del C. albifrons, que se extienden hasta el final de la misma. Son alargados y gruesos, en posición dorsal, lateral y ventral a lo largo de las vértebras caudales. Se identificaron los músculos sacrocaudal dorsal medial, sacrocaudal dorsal lateral, sacrocaudal ventral medial, sacrocaudal ventral lateral, intertrans-verso dorsal caudal, intertransverso ventral caudal, flexor caudal largo, extensor caudal lateral, coccígeo y elevador del ano. Estos músculos son más delgados y no se extienden hasta el final de la cola en el M. mullata. El músculo flexor caudal largo y el extensor caudal lateral han sido reportados en el M. mullata (Bast et al., 1961).

\section{Conclusiones}

- La descripción anatómica del miembro posterior y cola del Cebus albifrons es semejante a las descripciones realizadas para el mono Rhesus (Maccaca mullata).

- En la región de la pelvis y el muslo se observaron 18 músculos, pero no se encontró el músculo piriforme.

- En la región de la pierna, se observaron 12 músculos pero no se encontró el músculo peróneotibial y el peróneo digital del IV dedo.

- En la región del pie se observaron 11 músculos pero no se encontró el músculo oponente del I dedo.

- En la cola se observaron 10 músculos, cantidad superior con respecto a otros primates.

\section{Literatura Citada}

1. Ankel-Simons F. 2000. Primate anatomy: An introduction. $2^{\text {nd }}$ ed. New York, USA: Academic Press. 506 p.

2. Arsuaga J, Martínez L. 1998. La especie elegida. $2^{\mathrm{a}}$ ed. Madrid, España: Temas de Hoy. 9 p. 
3. Aversi TA, da Silveira PH, Aversi RAG, Silva Z. 2004. Anatomia do musculos flexores superficiales do antebrazo do macaco cebus. En: Reunión de Integración de la Morfología Panamericana. Foz de Iguazú, Paraná, Brasil. p 25.

4. Bast TH, Christensen K, Cummins H, Geist F, Hartman C, Hines M, Howell AB, Huber E. et al. 1961. The anatomy of the Rhesus monkey (Macaca mulatta). $2^{\text {nd }}$ ed. New York, USA: Hafner Publishing. 383 p.

5. Fortman JDT, Hewtt A, Bennet BT. 2002. The laboratory: Non human primate. $2^{\text {nd }}$ ed. New York, USA: CRC Press. 292 p.

6. Frey H. 1993. Der musculus triceps surae in der Primatenreihe. Morphologische Jahrbuch 47: 1-192.

7. Hershkovitz P. 1977. Living New World Monkeys (Platyrrhini) with an introduction to primates. Vol 1. Chicago,
USA: University of Chicago Press. $1164 \mathrm{p}$.

8. Hill W. 1960. Cebidae Part A. In: Primates: Comparative anatomy and taxonomy. London: Edinburgh University Press. 353 p.

9. [ICVGAN] International Committee on Veterinary Gross Anatomical Nomenclature. 2005. Nómina anatómica veterinaria. $5^{\text {th }}$ ed. Hannover: Editorial Committee. $166 \mathrm{p}$.

10. Jones BD, Eudey A, Geissmann T, Groves CP, Melnick DJ, Morales JC, Shekelle M, Stewart CB. 2004. Asian primate classification. Int J Primat 25: 97-164.

11. O'Rahilly R. 1989. Anatomía humana. $5^{a}$ ed. México DF: Interamericana McGraw-Hill. 928 p.

12. Shuck $A$. 1963. Beitrage zur myologyc der primaten. Der M latissimus dorsi und der M latissimus-tricipitalis. Morphologische Jahrbusch Morhp 45: 267. 